\title{
Protective Effect of Homogeneous Polysaccharide from Wuguchong(HPW) on Intestinal Mucositis Induced by 5- Fluorouracil in Mice
}

Peng Shi ( $\sim$ ilovetoto@126.com )

Dalian Medical University

Tianqi Zhao

Dalian Medical University

Wendong Wang

Dalian Medical University

Fangli Peng

Dalian Medical University

Ting Wang

Dalian Medical University

Yong Jia

Dalian Medical University

Linxuan Zou

Dalian Medical University

Peng Wang

Dalian Medical University

Simengge Yang

Dalian Medical University

Yue Fan

Dalian Medical University

Junwei Zong

First Affiliated Hospital of Dalian Medical University

Xueling Qu

Women's and Children's Hospital

Shouyu Wang

First Affiliated Hospital of Dalian Medical University https://orcid.org/0000-0002-2808-0247

\section{Research Article}

Keywords: Drug side effects, intestinal mucositis, Polysaccharide, Wuguchong, Intestinal barrier, 5Fluorouracil 
Posted Date: January 18th, 2022

DOI: https://doi.org/10.21203/rs.3.rs-1194334/v1

License: (c) (i) This work is licensed under a Creative Commons Attribution 4.0 International License. Read Full License 


\title{
Protective effect of homogeneous polysaccharide from
}

\section{Wuguchong(HPW) on intestinal mucositis induced by 5-}

\section{fluorouracil in mice}

Peng Shi ${ }^{1,2 \dagger}$, Tianqi Zhao ${ }^{1,2^{\dagger}}$, Wendong Wang ${ }^{6}$, Fangli Peng ${ }^{1,2}$, Ting Wang ${ }^{1,2}$, Yong Jia ${ }^{1,2}$, Linxuan Zou ${ }^{1,5}$, Peng Wang ${ }^{1,2}$, Simengge Yang ${ }^{1}$, Yue Fan ${ }^{1}$, Junwei Zong ${ }^{1,2 *}$, Xueling $\mathrm{Qu}^{3,4 *}$ and Shouyu Wang ${ }^{1,2 *}$

*Correspondence:aweizone@163.com;quxuelingmm@sina.com;wangshouyu666@12 $\underline{6 . c o m}$

$\uparrow$ Peng Shi and Tianqi Zhao contributed equally to this work.

1 Department of Orthopedic Surgery, The First Affiliated Hospital of Dalian Medical University, 222 Zhongshan Road, Dalian, China

2 College of Integrative Medicine, Dalian Medical University, 9 South Lushun Road West, Dalian, China.

3 Pelvic floor repair center, the Affiliated Dalian Maternity Hospital of Dalian Medical University, 1 Dunhuang Road, Dalian, China.

4 Pelvic floor repair center, Dalian women and Children Medical Center (Group), No.1

Road of Sports New Town, Dalian, China

5 Dalian Runxi Technology Development Co., Ltd, 3 Jinxia Street, Dalian, China

6 Department of Orthopedics, The Second People's Hospital of Dalian, 29 Hongji Street, Dalian, China

\begin{abstract}
Background: In hospitalized patients, drug side effects usually trigger off intestinal mucositis(IM), which in turn abates intestinal absorption and reduces the efficacy of treatment. It has been discovered that natural polysaccharides can relieve intestinal mucositis. In this study, we extracted and purified a homogenous polysaccharide of Wuguchong(HPW), one of traditional Chinese medicine, and explored the protective effect of HPW on 5-fluorouracil-induced IM.

Methods and Results: Firstly, we identified the physical and chemical properties of the extracted homogeneous polysaccharides. The molecular weight of HPW was 616 $\mathrm{kDa}$, which was composed of 14 monosaccharides. Then, a model of small intestinal mucositis induced by $5-\mathrm{FU}(50 \mathrm{mg} / \mathrm{kg})$ was constructed in mice to explore the effect and mechanism of HPW. The results showed that HPW effectively increased histological indicators such as villus height, crypt depth and goblet cell count. Meanwhile, it relieved intestinal barrier indicators such as D-LAC and DAO. Subsequently, western blot was used to detect the expression of Claudin-1, Occludin, PCNA, and inflammatory proteins such as NF- $\mathrm{B}(\mathrm{P} 65)$, TNF- $\alpha$, and COX-2. The figures also indicated that HPW could reduce inflammation and protect the barrier at the molecular level. Finally, we also investigated the influence of HPW on the content of SCFAs in feces of mice, a metabolite of intestinal flora.
\end{abstract}


Conclusions: HPW, as a bioactive polysaccharide derived from insects, has protective effects on intestinal mucosa and can relieve intestinal inflammation caused by drug side effects, which deserves further development and research.

\section{Keywords}

Drug side effects, intestinal mucositis, Polysaccharide, Wuguchong, Intestinal barrier, 5-Fluorouracil

\section{Background}

About $30 \%$ of inhospital patients are at exposure of malnutrition [1], which is mostly caused by reduced food intake, malabsorption, loss of gastrointestinal nutrients or increased energy expenditure.One important reason for this is malabsorption, which is associated with loss of epithelial integrity and impaired epithelial transport in the gastrointestinal tract [2].This pathological process not only occurs in the process of disease development, but also can be caused by drug side effects. For example, according to research statistics, $40 \%$ of cancer patients receiving chemotherapy develop lower gastrointestinal mucositis [3], which leads to malnutrition.

5-fluorouracil is an anti-metabolic anticancer drug, which is widely used in the treatment of cancer. However, a large proportion of patients using 5-fluorouracil will develop intestinal mucositis (IM) [4].Experimental studies have shown that 5-FU could decrease crypt and villi length by triggering apoptosis in intestinal epithelial cells [5].In addition, after 2 days of 5-FU treatment, NF- $\kappa \mathrm{B}$ transcription factor was highly overactive in the small intestine. Various inflammatory mediators including TNF- $\alpha$ are involved in the whole process of intestinal mucositis [6].Furthermore, among the molecular events that cause intestinal mucosal inflammation, chemotherapeutic drugs reduce the level of commensal bacteria, generating an imbalance of intestinal flora, which in turn leads to intestinal mechanical barrier and mucosal barrier dysfunction[7].

The treatment of small intestinal mucositis remains a difficulty and the traditional zinc derivatives, loperamide or mesalazine do not have desired effectiveness[8].Therefore, to search for natural producions capable of treating small intestinal mucositis is essential to protect against gastrointestinal damage and reduce inflammation.It has been investigated that natural dietary polysaccharides can improve small intestinal mucositis by promoting the development of epithelial cells and mucosal immune cells, enhance intestinal barrier function, and thus promote nutrient absorption. For example, the homogenous polysaccharide extracted from Dendrobium huoshanense has a regulatory effect on both intestinal and systemic immunity, and it can improve intestinal mucositis by improving mucosal barrier function and microbial composition in different regions of the intestine [9]. Sea cucumber fucoidan (SC-FUC) improves intestinal tissue architecture including indicators such as villi height and crypt depth, and ameliorates immune imbalance by regulating the $\mathrm{Th} 1 / \mathrm{Th} 2$ ratio to counteract small intestinal mucosal damage [10].However, the biological activity of insect polysaccharides has not been properly discovered compared with many natural organisms such as plants, fungi and marine organisms.Previously, glycosaminoglycan from dung beetle displayed anti-cancer properties and Huechys sanguinea 
glycosaminoglycan has been used in tuberculous amenorrhea and scabies[11].A novel polysaccharide extracted from the larvae of Black Soldier Fly (BSF) (Hermetia illucens) acts as an immune activator by stimulating the innate immune system of RAW264.7 cells through the TLR signaling pathway[12].

The traditional Chinese medicine Wuguchong is a kind of natural insect medicine produced by maggots, it is the dried larva of chrysomya megacephala or other related insects of calliphoridae. Ancient Chinese medicine books recorded its flavor is salty, sweet and its nature is cold. It has the function of invigorating spleen and eliminating food accumulation, clearing heat and eliminating infantile malnutrition. Previous studies have found that polysaccharide extracted from WuGuChong (PEW) could be used as a bioactive ingredient to prevent obesity[13]. Meanwhile, in this study, we tentatively explored the role of PEW in regulating intestinal microbial composition and maintaining intestinal epithelial integrity, which can reduce the ratio of Firmicutes to Bacteroides and the relative abundance of Proteobacteria in high-fat fed mice, and improve the expression of tight junction proteins. On the other hand, fatty acid extract of Wuguchong has been proved to promote wound healing on the surface of the body and promote the proliferation and migration of endothelial cells[14].

In this study, the polysaccharides were further purified and homogeneous polysaccharide of Wuguchong(HPW) was obtained by gel column chromatography, and its protective effect on intestinal mucositis induced by 5-fluorouracil was studied in mice.HPW protects the mechanical and immune barriers of the small intestine by improving the morphology of small intestinal villi, promoting goblet cell proliferation and tight junction protein expression.In addition, in the current study, we also assessed the amount of short-chain fatty acids (SCFAs) in the feces of mice.Based on previous studies[13], we speculated that HPW may protect chemotherapeutic drug-induced intestinal mucosal damage through intestinal flora and metabolic pathways, so as to achieve the function of promoting nutrient absorption.

\section{Methods}

\section{Extraction of HPW}

The crude extract of polysaccharides from Wuguchong was prepared by water extraction and alcohol precipitation according to our previous method [13].Simply put, dried insect powder is boiled three times in $95 \%$ alcohol in a Soxhlet reflux machine to remove excess lipids. Solid-liquid ratio of $20 \mathrm{mg} / \mathrm{L}, 110^{\circ} \mathrm{C}$, kept slightly boiling, coaqueous extraction for $6 \mathrm{~h}$. The concentrated solution was precipitated with 3 times ethanol for 2-3 days, the supernatant was poured, frozen and dried into powder, and the crude polysaccharide extract was obtained.

After protein removal by Sevage method, the crude extract was separated and purified by DEAE-cellulose column chromatography method in order to obtain homogenous polysaccharide with similar molecular weight and the same polarity. DEAE Sepharose Fast Flow packing was eluted with distilled water to neutral, the Flow rate was adjusted to $5 \mathrm{~mL} / \mathrm{min}$, and the balance was performed for $2 \mathrm{~h}$. The crude polysaccharides were dissolved in distilled water for elution, followed by step-wise elution with distilled water and $\mathrm{NaCl}$ solutions of $0.2,0.5$ and $2.0 \mathrm{M}$ at a flow rate of 15 
$\mathrm{mL} / \mathrm{min}$. Phenol-sulfuric acid method was used for tracking and detection. The microplate tester was used for detection at $490 \mathrm{~nm}$ and the scatter diagram was drawn (Figure 1A).

According to the peak shape, each component was collected, concentrated, dialyzed in a $3.5 \mathrm{kDA}$ molecular weight cut-off membrane, and freeze-dried . The fractions eluted with $0.2 \mathrm{M} \mathrm{NaCl}$ were further purified on a Sephacryl S-200 column $(1.6 \times 80 \mathrm{~cm})$ with the elution of potassium phosphate buffer (PBS, $0.1 \mathrm{M}, \mathrm{pH} 7.2)$ at a flow rate of $0.5 \mathrm{~mL} / \mathrm{min}[15]$.

Finally, the component with relatively high concentration was obtained, which was named as homogeneous polysaccharide of Wuguchong (HPW). The extraction rate was $1.2 \%$, and the purity was $87 \%$ by phenol-sulfuric acid method.

\section{Characterization and monosaccharide composition of HPW}

The molecular weight and purity of HPW was assayed with high performance gel permeation chromatography (HPGPC) (Shimadzu, Japan) using dextrans (MWs-5 kDa, $12 \mathrm{kDa}, 25 \mathrm{kDa}, 50 \mathrm{kDa}, 80 \mathrm{kDa}, 150 \mathrm{kDa}, 270 \mathrm{kDa}$, Sigma-Aldrich, USA) as standard samples for the TSK-gel GMPWXL $(7.8 \mathrm{~mm} \times 300 \mathrm{~mm}, 5 \mu \mathrm{m})$ [16]. Data showed that the weight-average molecular weight of the HPW was about $616 \mathrm{kDa}$, the purity was 87\%.(Table 1.)

High performance anion-exchange chromatography (HPAEC) was used to identify the monosaccharide composition of HPW.The chromatographic system used Thermo ICS5000 ion chromatography system (Thermo Fisher Scientific, USA), using electrochemical detector to analyze and detect monosaccharide components.Flow rate, $0.5 \mathrm{~mL} / \mathrm{min}$; injection volume, $5 \mu \mathrm{L}$; solvent system, $0.1 \mathrm{M} \mathrm{NaOH}:(0.1 \mathrm{M} \mathrm{NaOH}, 0.2 \mathrm{M}$ $\mathrm{NaAc}$ ); gradient program, 95:5 V/V at $0 \mathrm{~min}, 80: 20 \mathrm{~V} / \mathrm{V}$ at $30 \mathrm{~min}, 60: 40 \mathrm{~V} / \mathrm{V}$ at 30.1 $\mathrm{min}, 60: 40 \mathrm{~V} / \mathrm{V}$ at $45 \mathrm{~min}, 95: 5 \mathrm{~V} / \mathrm{V}$ at $45.1 \mathrm{~min}, 95: 5 \mathrm{~V} / \mathrm{V}$ at $60 \mathrm{~min}$.

The data showed that HPW was mainly composed of galactose $(21.55 \%)$, glucose $(20.77 \%)$, rhamnose $(7.05 \%)$, mannose $(7 \%)$, arabinose $(5.02 \%)$ and xylose $(3.21 \%)$. Ion chromatograph chart of samples shown in figure $1 \mathrm{~B}$, and the composition of monosaccharides is detailed in figure $1 \mathrm{C}$.

\section{Animals}

SPF grade C57BL/6 male mice weighing 18-22g and aged 6-8 weeks were purchased from the Experimental Animal Center of Dalian Medical University, China. All mice were fed adaptively for 7 days for subsequent operations. The ambient temperature was $20-25^{\circ} \mathrm{C}$ and the relative humidity was $40-60 \%$. Mice were fed with 12-h light - dark cycle, and were randomly given feed and drinking water. The procedures for animal experiments are strictly in accordance with the standard guidelines for laboratory animals and approved by the ethics committee of Dalian Medical University (Ethical Approval number: AEE19075).

\section{Experimental procedure}

Forty C57BL/6 male mice were randomly allocated into 5 groups with 8 mice in each group. The grouping is as follows:

Group 1: Water + Saline. 
Group 2: HPW(100 mg/kg BW $)+$ Saline.

Group 3: Water + 5-FU(50 mg/kg BW).

Group 4: HPW(100 mg/kg BW)+ 5-FU(50 mg/kg BW).

Group 5: Mesalazine (10 mg/kg BW)+ 5-FU(50 mg/kg BW).

In order to obtain a stable experimental animal model of intestinal mucositis, we adopted the protocol reported in previous studies[17]:normal saline and 5fluorouracil $(50 \mathrm{mg} / \mathrm{kg}$ ) were injected intraperitoneally in the first three days. Water, HPW or mesalazine was administered orally 1 hour before 5-FU administration for one week.

\section{Physical manifestations and tissue collection}

Body weight and diarrhea score of each mouse and food intake of each group were recorded on everyday of the experiment. Diarrhea severity was scored daily by a uninformed researcher based on criteria mentioned in previous studies[18]. The scoring criteria for diarrhea severity are as follows: 0:normal stool;1: slight(wettish and soft stool); 2: moderate(unformed stool, wet crissum and stained coat); 3: severe (watery stool)

At the end of the experiment ( 7 days after treatment), fresh feces particles of mice were collected and quickly put into liquid nitrogen for preservation and used for short-chain fatty acid (SCFA) detection. Mice were deprived of water and food for 12 hours. Blood was collected after pentobarbital anesthesia for enzyme linked immunosorbent assay (ELISA).The whole small intestine was rapidly dissected. A $2 \mathrm{~cm}$ intestinal segment of jejunum was taken at $15 \mathrm{~cm}$ behind the pylorus and fixed with $4 \%$ paraformaldehyde for hematoxylin-eosin (HE) staining and Periodic acid-Schiff (PAS) staining. The rest of the small intestine was used for other molecular biological experiments.

\section{Histological detection}

The 2-cm jejunum segment obtained from animal experiments was divided into two portions and laied up in 4\% paraformaldehyde fixative and Karnovsky fixative respectively. After paraformaldehyde fixation for 12-24 hours, the tissues were subjected to procedures such as dehydration and paraffin embedding. The slices with thickness of $5 \mu \mathrm{m}$ were cut and rehydrated with graded ethanol after deaffinity with xylene. Hematoxylin and eosin staining was then performed, and the dye was washed off with water, and the slices were dehydrated and sealed. Villus length and crypt depth, which are specific indicators of intestinal barrier and absorption function, were measured under a microscope (Olympus BX-40, Japan) using Image Pro Plus 6.0 software.

The other part of jejunal tissue was fixed in Karnovsky buffer and then similarly processed by dehydration, embedding and sectioning procedures. Sections were stained with Schiff's reagent for 20 minutes, followed by hematoxylin for 20 minutes.

The staining solution was washed away and the slices were subsequently dehydrated and sealed. The quantity of goblet cells on each villi was counted by Image Pro Plus 6.0 software. Goblet cells appear purplish red under microscope and are an important indicator of small intestinal mucosal barrier. Reagents were provided by Wuhan 
Servicebio Biotechnology Co., LTD.

\section{Reagents and antibodies}

Diamine oxidase activity(DAO) detection kit was purchased from Beijing Solarbio Technology Co., LTD. Total antioxidant capacity (T-AOC) test kit were obtained from Nanjing Jiancheng Biological Engineering Research Institute Co., LTD.ELISA kits (Shanghai Langton Biotechnology Co., LTD. )were used to measure D-lactate, a mechanical barrier marker, and SIgA, a mucosal barrier marker, respectively.Claudin-1, proliferating cell nuclear antigen (PCNA), tumor necrosis factor- $\alpha($ TNF- $\alpha)$ and GAPDH antibodies were provided by Wuhan Proteintech Biotechnology Co., LTD. Occludin, NF- $\mathrm{kB}(\mathrm{P} 65)$ and COX-2 antibody was from Abcam(Cambridge Science Park in Cambridge, UK).

\section{Western blot process}

The expression of Claudin-1, Occludin, PCNA, TNF- $\alpha, \mathrm{NF}-\kappa \mathrm{B}(\mathrm{P} 65)$ and COX-2 in jejunum and ileum tissue samples was detected by Western Blot. Tissue samples were lysed with RIPA buffer containing protease and phosphatase inhibitors to obtain total protein , followed by BCA protein quantification. Related reagents were purchased from Sevenbio (Beijing) Co., LTD.The lysed supernatant (about $80 \mu \mathrm{g}$ protein) was transferred to PVDF membrane (Millipore, USA) by 10\% SDS-PAGE.After two hours of blocking at room temperature with 5\% non-fat milk, the membranes were incubated with primary antibodies for $4{ }^{\circ} \mathrm{C}$ overnight: Claudin-1(1:1000), Occludin(1:2000),PCNA(1:5000), TNF- a (1:1000), NF-kB(p65) (1:1000),COX2(1:1000), GAPDH (1:2000).RP-conjugated rabbit anti-mouse IgG and goat anti-rabbit IgG were incubated with secondary antibodies at room temperature for $1 \mathrm{~h}$, and the protein bands were viewed with ECL detection system (Syngene, UK).Image Pro Plus 6.0 software was used to analyze gray values.

\section{Measurement of SCFAs}

The content of SCFAs in mouse fecal samples was measured according to a previously reported method[19].Take an appropriate amount of the sample, add $0.3 \mathrm{~mL}$ water, $100 \mu \mathrm{L} 50 \%$ sulfuric acid, $25 \mu \mathrm{L} 500 \mathrm{mg} / \mathrm{L}$ internal standard (cyclohexanone) solution and $0.5 \mathrm{~mL}$ ether homogenate for $1 \mathrm{~min}$, centrifuge at $4{ }^{\circ} \mathrm{C} 12000 \mathrm{rpm}$ for $10 \mathrm{~min}$, take the supernatant and put it on the machine for test(Gas Chromatohraph-Mass Spectrum, Shimadzu GCMS QP2010-Ultra, Japan). Chromatographic system: Agilent DB-WAX capillary column $(30 \mathrm{~m} \times 0.25 \mathrm{~mm} \times 0.25 \mu \mathrm{m})$; The carrier gas is high purity helium $\left(\geqslant 99.9 \%\right.$ ), and the flow rate is $1.0 \mathrm{~mL} / \mathrm{min}$. The inlet temperature is $220^{\circ} \mathrm{C}$, the injection volume is $1 \mu \mathrm{L}$, and the solvent delay time is $2.5 \mathrm{~min}$, splitless injection. Mass spectrometry system: Electron bombardment ion source (EI), ion source temperature is at $230{ }^{\circ} \mathrm{C}$, interface temperature is at $220^{\circ} \mathrm{C}$. The chromatograph is connected to a microcomputer instrumented with a detector for outputting the results of chromatographic analysis with the GC Solution program (Shimadzu, Japan).

\section{Statistical analysis}


Data are presented as means \pm SEM, and analyzed using the one-way ANOVA with GraphPad Prism 8.0 followed by Dunnett's test. We considered the data significant when $\mathrm{P}<0.05$.

\section{Results}

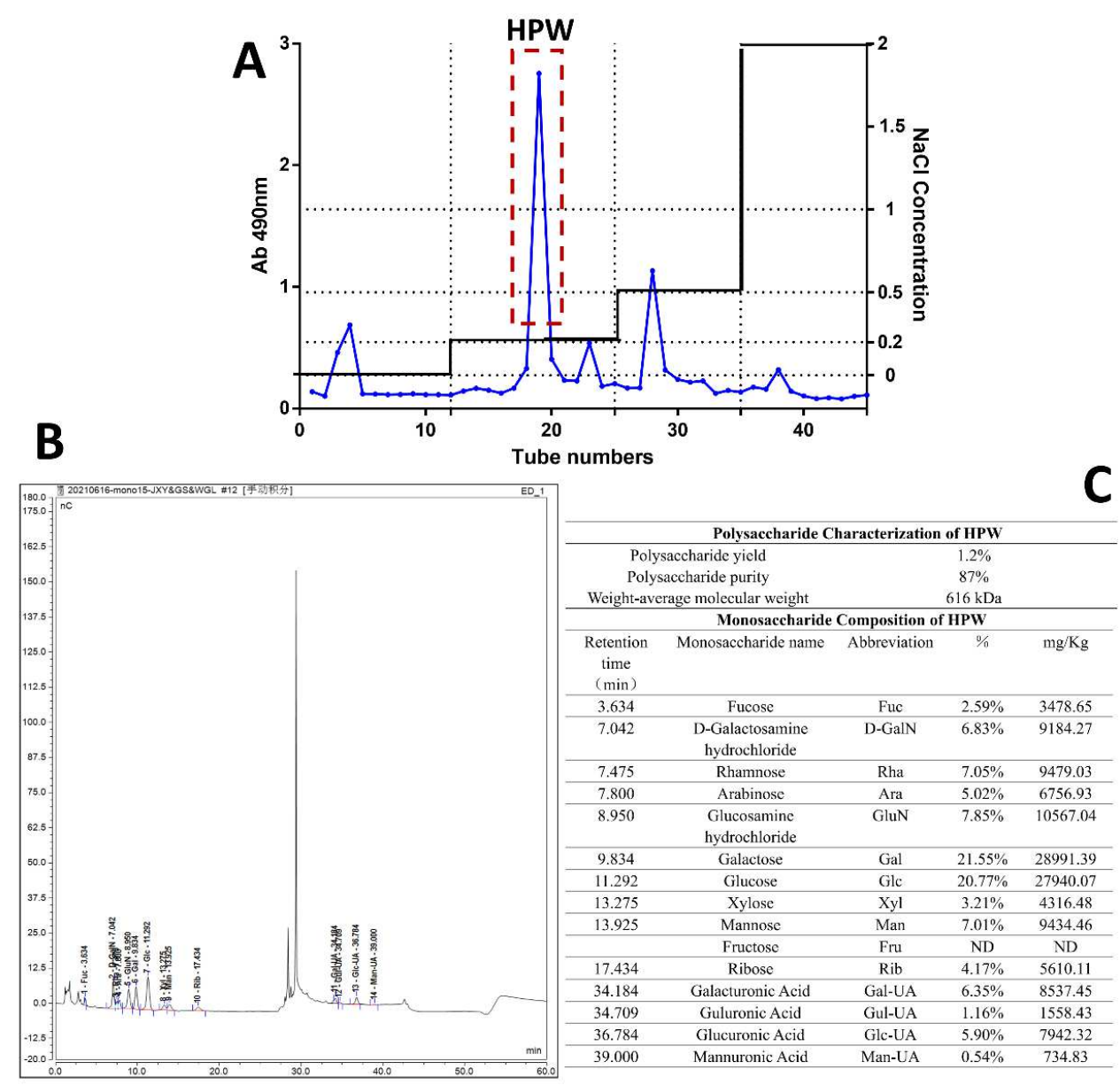

Fig. 1 Purification and identification of homogenous polysaccharide of Wuguchong(HPW). (A) Elution curves of crude polysaccharides and the position of HPW. The abscissa is the number of elution tubes, and the ordinate is the absorbance value of tracking sugar content under detection at $490 \mathrm{~nm}$ by phenol sulfuric acid method. (B) Ion chromatograph chart of monosaccharide composition by HPW samples. Abscissa is the retention time of detection (Time, min), and ordinate is the response value of ion detection (Response, nC). (C) Aggregated table of properties and monosaccharide composition of HPW. 

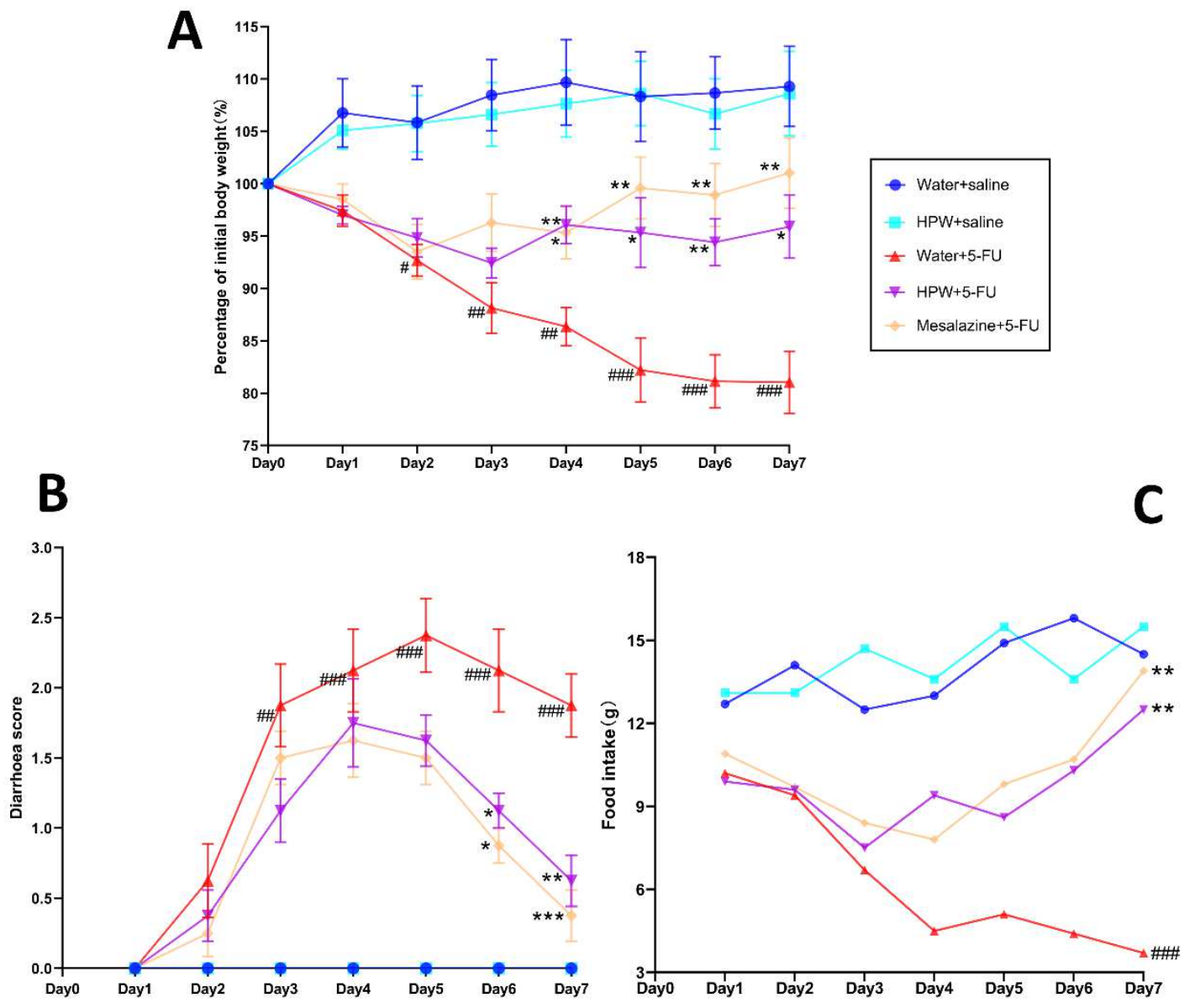

Fig. 2 Effect of HPW on physiological manifestations of mice. (A) Body weight change analysis. (Weight/initial weight) $\times 100 \%$. (B) The diarrhea score of the experiment mice. (C) Food intake of mice in each group. Data are presented as means \pm SEM, and analyzed using the one-way ANOVA followed by Dunnett's test $(n=8)$. The “*”represents the comparison with the model group(Water+5-FU) and “\#”represents the comparison with the control group(Water + Saline). One tag means $\mathrm{p}<0.05$, two tokens represent $\mathrm{p}<0.01$, and three is $\mathrm{p}<0.001$.

\section{Effect of HPW on physiological manifestations of mice}

Body weight change, diarrhea index, and food intake are important phenotypic indicators of intestinal mucosal inflammation.In this present study, there was no significant weight loss, decline of food intake, diarrhea, or death were observed the control group (water + saline).Compared with the control group, the modeling group (water +5 -FU) experienced significant weight loss from day 2 and 3, accompanied by diarrhea (unformed or even watery stools) and reduced food intake (Figure 2).However, after HPW intervention, the above situation improved. From the fourth day onwards, weight loss (Figure 2A) and diarrhea score (Figure 2B) were relieved in the HPW+5FU group, and food intake gradually resumed (Figure $2 \mathrm{C}$ ). Therefore, we speculated 
that HPW has a protective effect on intestinal mucositis .At the same time, we also evaluated the physiological status of mice given HPW alone by intragastric administration. As shown in Figure 2, there was no statistical difference between the HPW + saline group(the light blue curve) and the control group, indicating that the supplement of HPW alone would not have adverse effects. In this part of the experiment, we took mesalazine as a positive control, and the physical status of mice in mesalazine +5-FU group was improved as anticipated.

\section{Effect of HPW on the histological status of small intestinal mucositis in mice.}

Figure 3A shows that the cross sections of the jejunum in fluorouracil induced group (Water $+5-\mathrm{FU}$ ) displayed severely damaged pathological structure, showing villus height decreased significantly, crypt depth decreased and morphological dysplasia. Vacuolar edema and inflammatory cell infiltration were observed in the submucosa and muscularis. HPW and mesalazine treatment alleviated the histopathological damage in different degrees. The intestinal villus height, crypt depth, and villus crypt ratio were remarkably enhanced in the HPW $+5-\mathrm{FU}$ group (Figure 3B, $\mathrm{C}$ and D), demonstrating a protective effect against chemotherapeutic drug-induced mucosal injury. Compared with the control group, intragastric administration of HPW alone had no effect on small intestinal micromorphology.

\section{Analysis of goblet cell counting and sIgA secretion}

Compared with the Water + saline group, intraperitoneal injection of 5-FU markedly lowered the goblet cell count on each small intestinal villi (Figure 3E). After management with HPW and mesalazine, the cupped cell count returned and alignment returned to normal with statistically significant differences. There was no statistically significant difference between the Control group and the HPW treatment group alone (Figure 3F). The same trend was also reflected in the content of intestinal secreted IgA detected by ELISA, that is, fluorouracil drug intervention reduced the secretion of sIgA, and both mesalazine and HPW could be effectively reversed with significant difference (Figure 4C).

\section{Effects of HPW on the inflammation-associated proteins NF- $\mathrm{BB}, \mathrm{COX}-2$ and TNF- $\alpha$}

The effects of HPW on the expression level of intestinal NF-kB, COX-2 and TNF$\alpha$ are shown in Figure 5. Compared with the control group, the expressions of these three key targets in Water+5-FU group were significantly enhanced. This suggests activation of inflammatory pathways at the molecular level. HPW can reverse the negative levels caused by the side effects of 5-FU (Figure5A-C). 

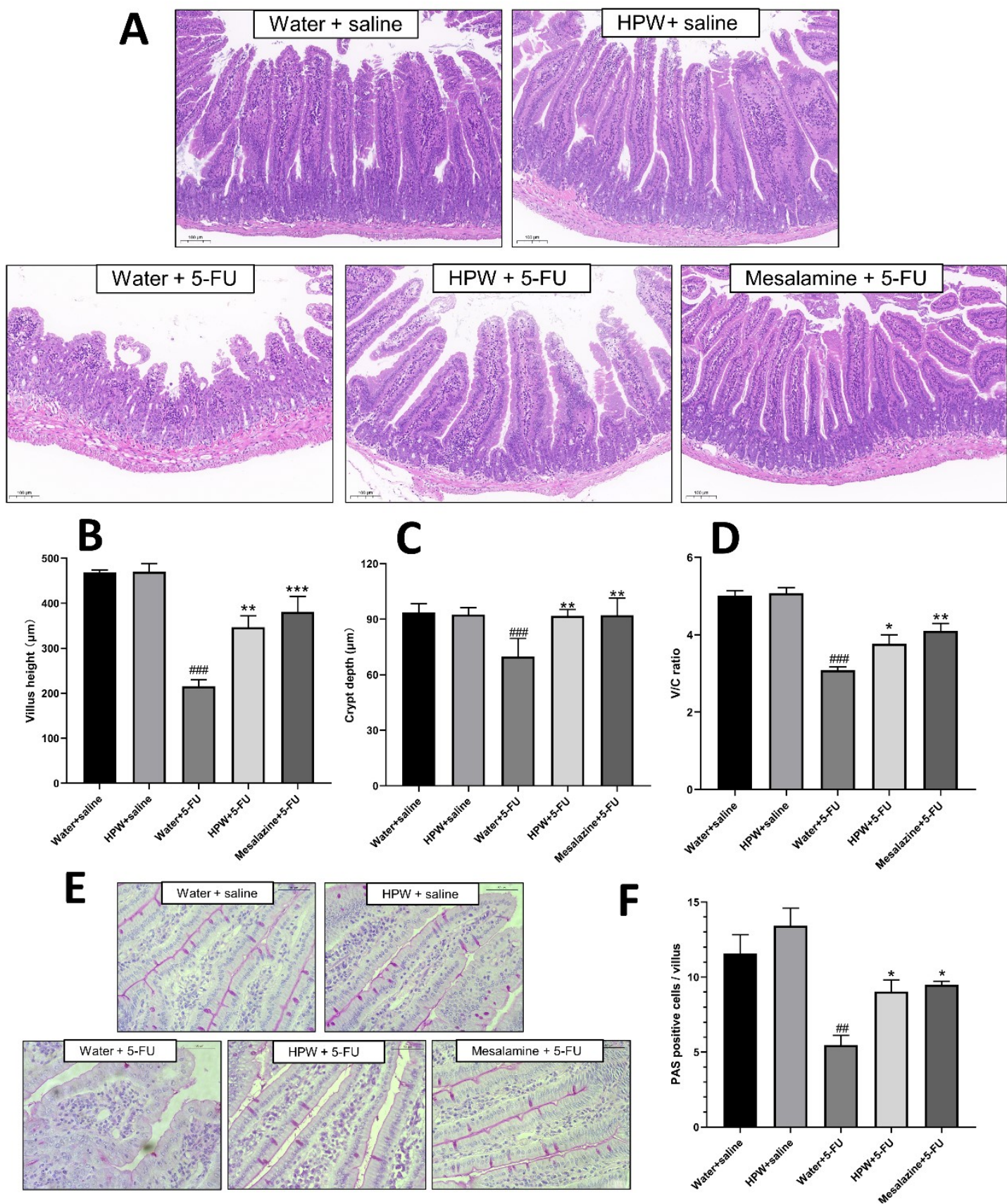

Fig. 3 Microhistological examination of small intestine. (A) HE staining of intestine tissues (scale bar=100 $\mu \mathrm{m}$ ). (B) villus height. (C) Crypt depth. (D) The ratio of V/C. (E) PAS staining of goblet cells (scale bar $=50 \mu \mathrm{m}$ ). (F) Analysis of goblet cell counting. Data are presented as means \pm SEM, and analyzed using the one-way ANOVA followed by Dunnett's test $(\mathrm{n}=8)$. The “**"represents the comparison with the model group(Water $+5-\mathrm{FU}$ ) and "\#" $\#$ "represents the comparison with the control group(Water + Saline). One tag means $p<0.05$,two tokens represent $p<0.01$, three is $p<0.001$. 

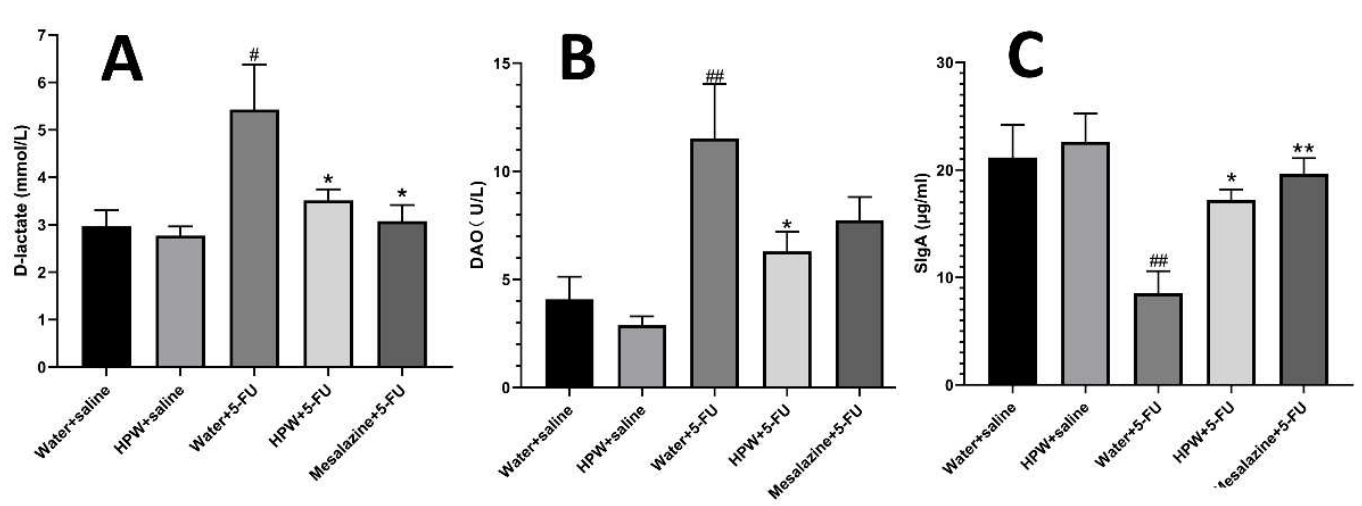

D
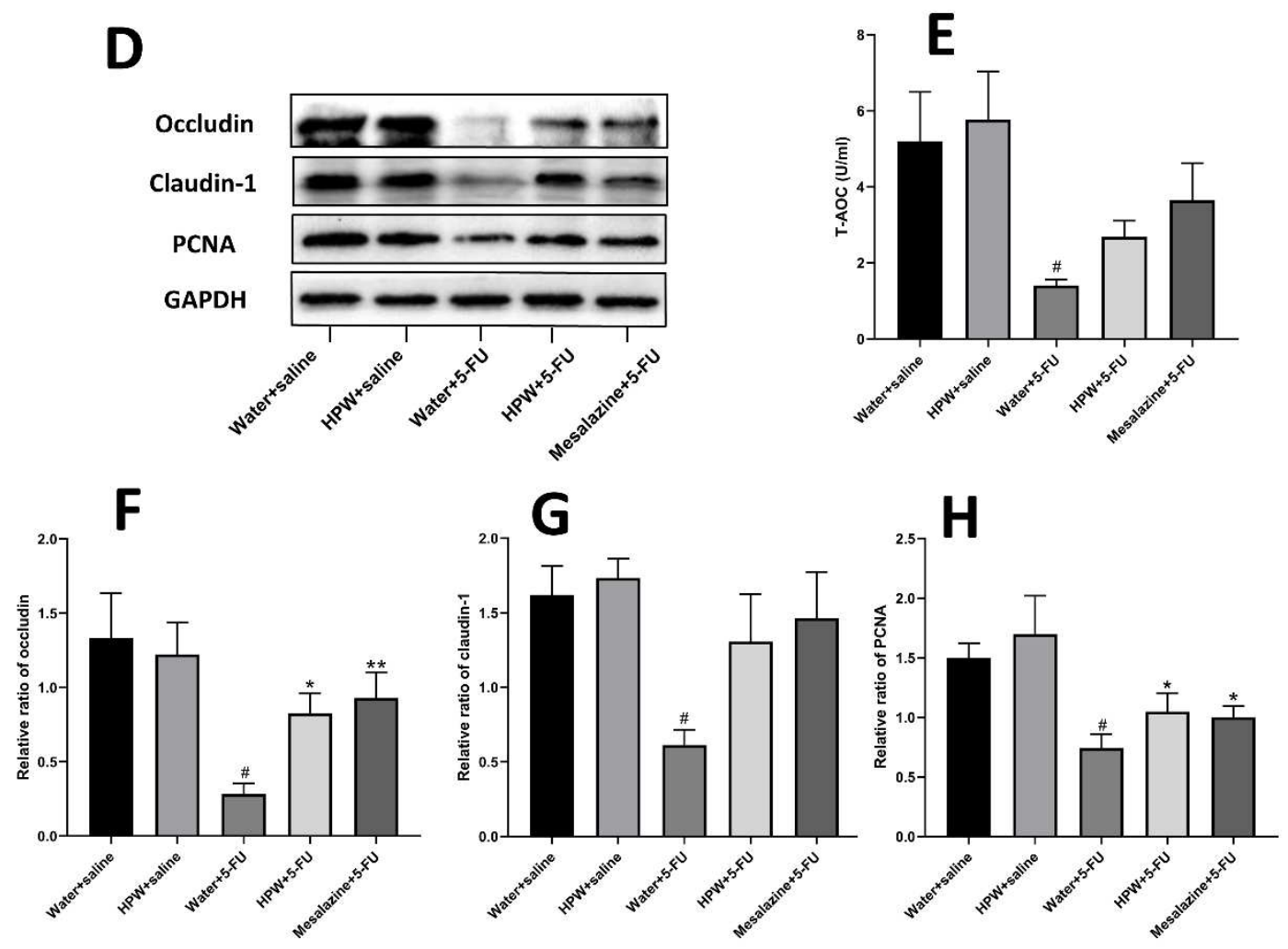

Fig. 4 Effect of HPW on small intestinal barrier indices. (A) D-lactic acid (D-LA); (B) diamine oxidase (DAO) (E) total antioxidant capacity (T-AOC) in serum. (C) SIgA in tissue homogenate. (D) Contrast diagram of Occludin, Claudin-1, proliferating cell nuclear antigen (PCNA) and GAPDH protein protein bands. (F, G, H) Analysis of their respective grayscale values. Data are presented as means \pm SEM, and analyzed using the one-way ANOVA followed by Dunnett's test $(\mathrm{n}=8)$. The “*” represents the comparison with the model group(Water +5 -FU) and "\#"represents the comparison with the control group(Water + Saline). One tag means $\mathrm{p}<0.05$, two tokens represent $\mathrm{p}<0.01$, three is $\mathrm{p}<0.001$. 


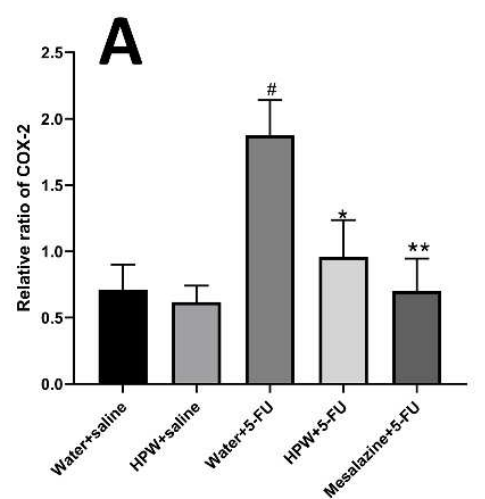

D

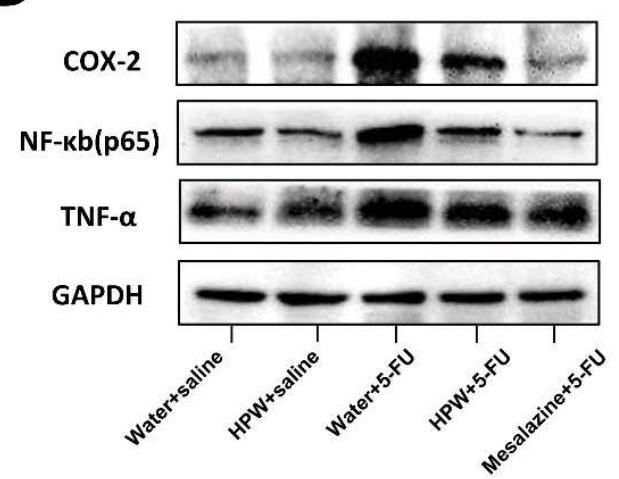

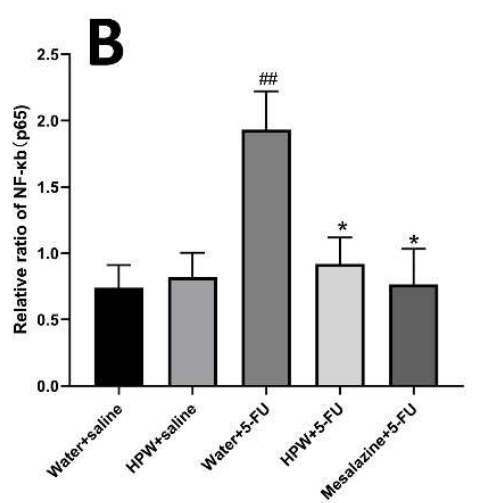

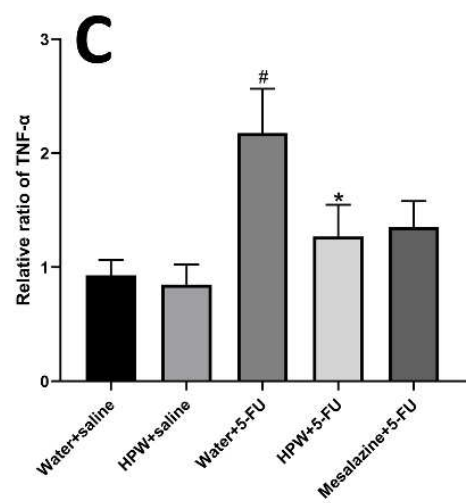

$\mathbf{E}$

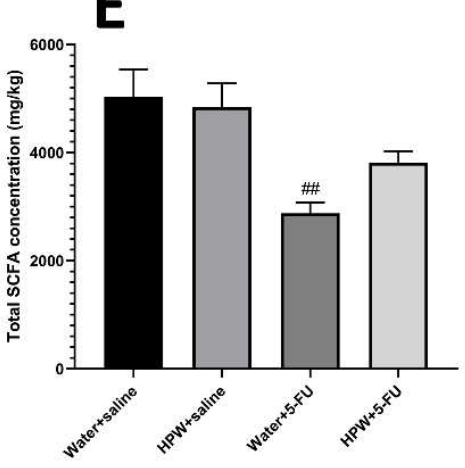

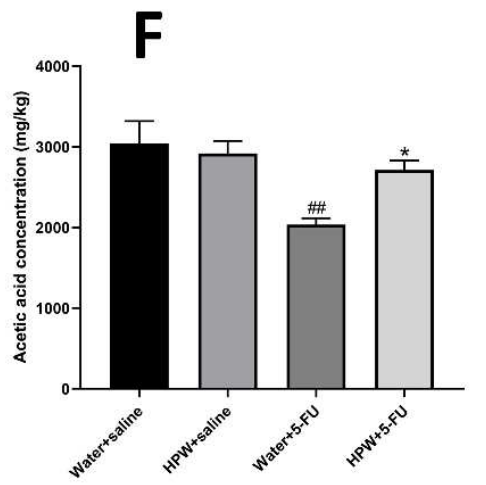
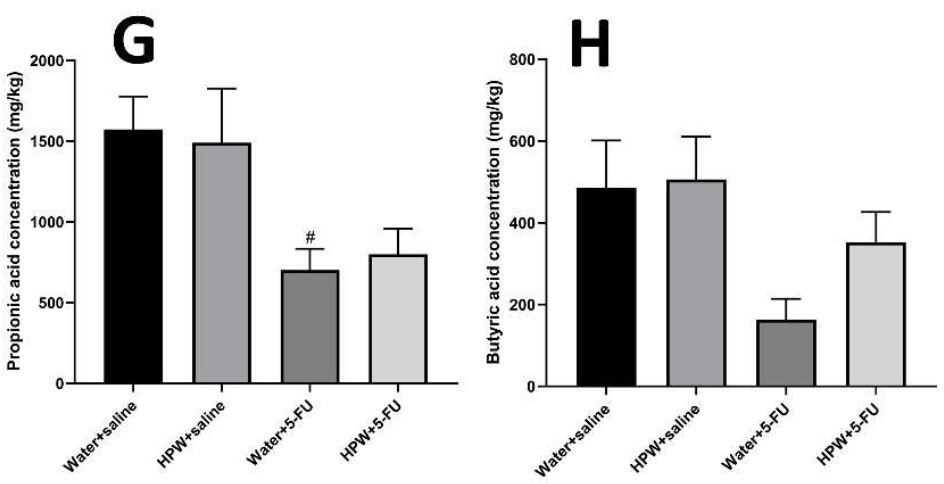

Fig. 5 Effect of HPW on inflammatory pathway and SCFAs. Grayscale values analysis of (A) COX-2; (B) NF-kB and (C) TNF- $\alpha$. (D) Contrast diagram of these three molecules and GAPDH protein bands. Analysis of (E) total short chain fatty acids(SCFAs); (F) acetic acid; (G) propionic acid; (H) butyric acid. Data are presented as means $\pm \mathrm{SEM}$, and analyzed using the one-way ANOVA followed by Dunnett's test $(\mathrm{n}=8)$. The "*" represents the comparison with the model group (Water $+5-\mathrm{FU})$ and “\#”represents the comparison with the control group(Water + Saline). One tag means $\mathrm{p}<0.05$, two tokens represent $\mathrm{p}<0.01$, three is $\mathrm{p}<0.001$.

\section{Analysis of short chain fatty acids (SCFAs)}

In our current data, figure $5 \mathrm{E}$ indicates the changes in total short-chain fatty acids: the content of fecal SCFAs in mice dropped after 5-FU treatment and increased after HPW supplementation. The same tendency appears in the assay of acetic acid content and is significant (Figure 5F). Consistent with the previous two indices, the addition of 5-Fu reduced the contents of propionic acid and butyric acid (Figure5 G, H). And yet, 
the former level (propionic acid, Figure 5 G) did not change after HPW gavage and the latter (butyric acid, Figure $5 \mathrm{H}$ ) was not statistically significant. In this part of the experiment, there was also no significant variation in the mice with HPW administered alone compared to the control group.

\section{Discussion}

It has been extensively demonstrated that the biological activity of polysaccharides is related to its own chemical characteristics, monosaccharide composition and the binding structure of glycosidic bonds[20]. Oral natural non-starch polysaccharides can be rapidly degraded into low-molecular weight polysaccharide fragments in the gastrointestinal tract, which are stably retained in the fluid of the stomach and small intestine to participate in the digestion and absorption process, and absorbed into the blood to participate in systemic circulation[21]. Our data show that there is $20.77 \%$ glucose in HPW, which is similar to many polysaccharides with intestinal mucosal protection.Glucose can be absorbed into the blood through the combination and internalization of intestinal epithelial cells, playing the role of anti-inflammatory and immune regulation[22].

The extent of intestinal injury induced by 5-FU was reported to be dose-dependent, with moderate weight loss and diarrhea in intraperitoneal mice injected at 50 and 100 $\mathrm{mg} / \mathrm{kg}$.At this dose range ,mice mortality was low and activity was stable[23]. In our experiment, typical pathogenic changes of intestinal mucositis in mice were observed by intraperitoneal injection of 5-fluorouracil $(50 \mathrm{mg} / \mathrm{kg}$ ) for three consecutive days (Day 1-Day 3), which was consistent with previous studies[24].Clinically, cancer patients lose weight and appetite, which is associated with nausea and vomiting associated with chemotherapy[25,26].Based on the above mouse model of intestinal mucositis, our experimental results showed that HPW could improve 5-FU-induced diarrhea and gradually restore body weight and food intake without adverse effects.

In the present study, intraperitoneal administration of 5-FU caused significant structural damage to the small intestine. Villus atrophy inevitably affects intestinal absorption, which may be partly responsible for weight loss, as shown in Figure 2A. HPW treatment significantly increased the villus height and crypt depth of jejunum and restored the morphology of villus and crypt; possibly representing HPW promoted crypt cell regeneration and hence and thus increased crypt cell migration to villi. Reconstruction of intestinal micromorphology promotes recovery of absorption function, which may lead to increased food intake as shown in Figure 2C.Other studies have indicated that the administration of Sijunzitang polysaccharides significantly reduced the evaluation of appearance and histopathological examination during delayed healing of gastrointestinal ulcers, a process associated with polysaccharide promoting crypt epithelial cell migration[27].In addition, the water soluble polysaccharides of rhubarb also play a role in protecting intestinal mucosal cells from apoptosis by mediating antioxidant effects and maintaining the structure of villi and crypts[28].Further investigations are therefore required to comprehend the protective mechanism of Wuguchong homogenous polysaccharides (HPW) in improving intestinal inflammation and damage to the mucosa. 
Goblet cells, a health marker of the intestinal mucosal epithelium and a secretory component of the intestinal mucosal barrier, are seen by a periodate - Schiff (PAS) stain[29]. A reduced count of these cells represents damage to the mucosal layer of the small intestine, exposing the epithelial surface of the intestinal lumen to bacterial translocations[30]. Goblet cells and other small intestinal mucosal epithelial cells together produce mucin covering the intestinal mucosal layer, which is similar to the mucus gel that protects the gastrointestinal tract and is a vital component of the mucosal barrier[31]. SIgA, a major component of mucin, is the principal protective molecule of specific (acquired) immunity secreted to mucosal surfaces, which optimizes microbial groups, prevents them from adhering to mucosal surfaces, and reduces the toxic expression of intestinal pathogens and effectively prevents bacterial translocation[32]. Our data suggested that HPW can significantly up-regulate the quantity of GCs and sIgA level, suggesting that Wuguchong polysaccharide can alleviate small intestinal mucosal inflammation by enhancing mucosal barrier function.

We found histologically that HPW alleviates 5-Fu-induced intestinal mucositis by improving impaired intestinal barrier function, and then provided biochemical evidence to support these results. DAO is an enzyme in intestinal epithelial cells that suppresses cell proliferation by reducing polyamine concentration, whereas D-lac is a bacterial metabolite produced by intestinal flora [33]. Basal levels of both are generally low in the normal mammalian systemic circulation and are usually observed only in the gut. During intestinal infection and inflammation, intestinal wall permeability increases and translocations of numerous microorganisms from the intestine to circulation increase, intraluminal dao d-lac easily enters peripheral blood through intestinal mucosa[34]. Moreover, the intestine is the largest contact surface between the human body and the external world, and is the central organ of the body's stress response under stressful conditions[35]. Chemotherapy enterotoxic drugs can attack the gastrointestinal tract with excessive free radicals, leading to impaired metabolism of intestinal epithelial cells, damaged cell function and inflammatory response[36]. Therefore, we also initially explored the oxidative stress-related indicator T-AOC. The results of our current study were consistent with those above that the serum level of DAO and D-LAC in the 5-Fuinduced mucositis mouse model was higher than that in the control group, indicating that the intestinal permeability of mice was expanded. The content of T-AOC was also greater than that of the control group, suggesting oxidative stress reaction.Meanwhile, HPW supplementation alleviated all three indicators, recommending that HPW may alleviate intestinal mucositis by reestablishing intestinal barrier function and lessening oxidative stress response.

Above, we discussed the mechanism of HPW alleviating intestinal mucositis from the aspects of intestinal mucosal barrier and permeability. However, intestinal homeostasis is a dynamic boundary between internal and external environments, and the a apical junctional complex constitute a crucial role in maintaining intestinal homeostasis, just like intestinal epithelial cells. These tight junction proteins, including the representative transmembrane proteins Claudins and Occludin, bind endothelial cells together by means of scaffold proteins and actin, forming the mechanical barrier of the intestinal tract [37]. Claudins prevent the unlimited flow of water and solutes, as 
well as the invasion of luminal antigens[38]. Interaction of claudins- 1 with integrin in local adhesion, involved in the regulation of transport between cells and the extracellular matrix. Claudins-1 molecule can regulate normal cell homeostasis under physiological conditions and promote the adhesion of migrated cells under pathological conditions[39, 40].Occludin, as the first tight junction protein discovered, is thought to regulate extracellular permeability by sealing adjacent cells [41].In vitro studies reported that restoration of Occludin high expression improved the molecular barrier function of pig intestinal epithelial cells (IPEC-J2) [42]. Previous investigation has confirmed that chemotherapy-induced intestinal barrier damage in IM mice is associated with the decrease expression of occludin[43].Our present experiment revealed a decrease expression of Claudin-1 and Occludin in intestinal of mice treated with 5-FU, while the expression of these two tight junction proteins recovered in mice fed with Wuguchong homogeneous polysaccharides. The results strengthen the relevant studies on the role of tight junction protein in intestinal barrier function, and confirm that HPW can indeed promote the recovery of intestinal mechanical barrier and improve intestinal mucositis by regulating intestinal tight junction protein.In addition, we also investigated the expression of PCNA. Proliferation cell nuclear antigen(PCNA) is involved in DNA replication and double DNA strand reconstruction. It is considered a signature of cell cycle dynamics and proliferative activity [44].Continuous proliferation of intestinal epithelial cells and subsequent enhancement of tissue recovery can attenuate intestinal inflammation[45]. Therefore, PCNA is a forward molecule for evaluating the intestinal epithelial barrier.In our current study, PCNA expression was reduced in the model-induced group and HPW expression was upregulated this indicator. It was further confirmed that the homogenous polysaccharide of Wuguchong could promote the recovery of intestinal barrier.

A better understanding of the molecular mechanism that lead to intestinal mucositis could provide therapeutic methods for curing these drug side effects and boosting absorption function of small intestine.In 2004, Sonis et al. proposed an overlapping five-step model to summarize the biological phases of mucositis : initiation, primary damage response, signal amplification, ulcer formation, and healing[46]. The formation of reactive oxygen species, inflammation, and cell apoptosis are the most important molecular events in the early stages of the injury.Among these sequential molecular events, NF- $\mathrm{BB}$ has been considered to be one of the most important transcription factors associated with tumor toxicity and therapeutic resistance[47]. Activation of NF- $\kappa$ B can upregulate more than 200 different genes, $m$ ny of these may have mucosal toxicity[48].TNF- $\alpha$ and COX-2, both of which are important target genes of NF- $\kappa B$ involved in immune response stress in inflammatory cascade[49]. Thus, we preliminarily estimated the expression of these three key molecules in the inflammatory NF- $\kappa$ B-COX-2-TNF- $\alpha$ pathway at the protein level.The data suggest that HPW can improve the molecular down-regulated expression caused by the side effects of 5-FU administration, which may be one of the molecular mechanisms by which it can improve the inflammation of the small intestinal mucosa.In addition, other studies have demonstrated that TNF- a may initiate the infiltration of inflammatory cells into the intestine by decreasing the expression of ocludin leading to structural changes in tight 
junctions[50]. COX-2 destroys the collagen subepithelial matrix and epithelial basement membrane by activating matrix metalloproteinases, which further damages the small intestinal mucosal barrier[51].

For the past few years, an increasing number of researches have confirmed that the intestinal microenvironment is a dynamic balance between organism and microbiota. The intestinal microbiota contains multiple carbohydrate active enzymes (CAZymes), which participate in the metabolism of dietary polysaccharides and produce SCFAs beneficial to host health $[52,53]$. Research shows that SCFAs (i.e. acetate, propionate, and butyrate) can be resorbed by intestinal epithelial cells and protect intestinal barrier[54]. In addition, SCFAs also can improve 5-FU-induced small intestinal mucosal inflammation by improving the intestinal mucosal barrier and reducing the level of inflammation [55]. Our results are in agreement with that of Flavia et al. [56] that 5-FU treatment reduced SCFAs content in mice feces. After HPW intervention, the content of total SCFAs, acetic acid and butyric acid increased, indicating that microbial activity gradually recovered. Some studies have shown that Bacteroides can participate in the metabolism of polysaccharides to form succinic acid, which can be used as a single carbon source to produce such as acetic acid and other SCFAs[57].In our previous study, it was found that supplementation with polysaccharide from Wuguchong could modify the obesity status of mice fed with high fat and high sugar by increasing the abundance of Bacteroidetes and decreasing that of Firmicutes[13].Therefore, we hypothesized that the beneficial interaction between HPW and Bacteroidetes in mice might be one of the reasons for the increase of SCFAs in the current study. Some intestinal probiotics can degrade xylose and glucose in polysaccharides into propionic acid [58]. However, there was no significant change in propionic acid content in our study. We guess that this is probably related to the fact that HPW comes from insects and has low xylose content (3.21\%, Figure 1C). Moreover, butyrate acts as one of health-promoting SCFAs, providing energy to epithelial cells, enhancing mucosal barrier function and reducing inflammation[59]. In present study, HPW was still found to improve butyrate content, but there was no statistical significance. It is probably related to the dose and time of our intervention, which is also the focus of our follow-up study.

\section{Conclusions}

Our results suggest that HPW, as a bioactive polysaccharide extracted from insects, has a palliative effect on intestinal mucositis caused by drug side effects. It can alleviate 5-FU-induced mechanical damage to the intestinal barrier in mice, improve the expression of tight junction protein, and reduce the activation of inflammatory pathways. At the same time, HPW can also promotes the content of short-chain fatty acids, which were metabolites of intestinal flora, and regulate intestinal microecology. In future researches, we will further purify this natural product and try to explore the mechanism of action in vitro.

\section{List of abbreviations}

HPW: homogeneous polysaccharide from Wuguchong; IM: intestinal mucositis; 
PCNA: proliferation cell nuclear antigen; D-LAC:D-lactate; DAO: diamine oxidase; SCFA: short-chain fatty acid; 5-FU:5-fluorouracil; HE: hematoxylin-eosin; PAS:

Periodic acid-Schiff; T-AOC: total antioxidant capacity;

GC-MS: Gas Chromatography-Mass Spectrometer

\section{Ethics approval and consent to participate}

The procedures for animal experiments are approved by the ethics committee of Dalian Medical University (Ethical Approval number: AEE19075).

\section{Consent for publication}

Not applicable.

\section{Availability of data and material}

The datasets used and/or analyzed during the current study are available from the corresponding author on reasonable request.

\section{Competing interests}

The authors declare that they have no competing interests.

\section{Funding}

This study was supported by National Natural Science Foundation of China (82074426,82104864,81573734), Natural Science Foundation of Liaoning Province (2021-BS-215), Liaoning Revitalization Talents Program (XLYC1802014), Liaoning Key Research and Development Planning Project (2017226015), Liaoning BaiQianWan Talents Program, Distinguished Professor Project of Liaoning Province, Dalian outstanding youth science and technology talent project(2015R003).

\section{Authors' contributions}

Junwei Zong, Xueling Qu and Shouyu Wang contributed to the study's conception and design. Peng Shi and Tianqi Zhao contributed to drafting of the manuscript. Peng Shi and Wendong Wang performed data analysis and interpretation. Tianqi Zhao, Fangli Peng and Ting Wang performed animal experiments. Yong Jia, Linxuan Zou, Peng Wang are responsible for drug extraction. Simengge Yang, Yue Fan and Peng Shi completed the molecular biology experiment. The final draft was read and approved by all the authors.

\section{Acknowledgements}

Not applicable.

\section{References}

[1] Felder S , Lechtenboehmer C , Bally M, et al. Association of nutritional risk and adverse medical outcomes across different medical inpatient populations[J]. Nutrition, 2015:1385-1393.

[2] Scaldaferri F , Pizzoferrato M , Lopetuso L R, et al. Nutrition and IBD: 
Malnutrition and/or Sarcopenia? A Practical Guide[J]. Gastroenterology Research \& Practice, 2017, 2017:8646495.

[3] Krishna S G, Zhao W , Grazziutti M L, et al. Incidence and risk factors for lower alimentary tract mucositis after 1529 courses of chemotherapy in a homogenous population of oncology patients: clinical and research implications.[J]. Cancer, 2011, 117(3).

[4]Small intestinal mucosal injury in patients with gastrointestinal cancer who develop complicated fluoropyrimidine-induced diarrhea. 2020 .

[5]Gastrointestinal dysmotility in 5-fluorouracil-induced intestinal mucositis outlasts inflammatory process resolution[J]. Cancer Chemotherapy \& Pharmacology, 2008, 63(1):91-98.

[6] Chang C T , Tin-Yun H, Lin H, et al. 5-Fluorouracil Induced Intestinal Mucositis via Nuclear Factor- $\kappa \mathrm{B}$ Activation by Transcriptomic Analysis and In Vivo Bioluminescence Imaging[J]. Plos One, 2012, 7(3):e31808.

[7] Tang Y, Wu Y, Huang Z, et al. Administration of probiotic mixture DM\#1 ameliorated 5-fluorouracil-induced intestinal mucositis and dysbiosis in rats[J]. Nutrition, 2016:96-104.

[8] Wardill H R , Bowen J M , Gibson R J . New pharmacotherapy options for chemotherapy-induced alimentary mucositis[J]. Expert Opinion on Biological Therapy, 2014, 14(3):347-54.

[9] Szx A , Bing L A, Hyy A, et al. Dendrobium huoshanense polysaccharide regionally regulates intestinal mucosal barrier function and intestinal microbiota in mice[J]. Carbohydrate Polymers, 2019, 206:149-162.

[10] Zuo T, Li X , Chang Y, et al. Dietary fucoidan of Acaudina molpadioides and its enzymatically degraded fragments could prevent intestinal mucositis induced by chemotherapy in mice[J]. Food \& Function, 2015, 6(2):415-422.

[11] Ahn M Y , BJ Kim, Kim H J , et al. Anti-cancer effect of dung beetle glycosaminoglycans on melanoma[J]. BMC Cancer, 2019, 19(1).

[12] MF Z Ali, Ohta T, Ido A, et al. The Dipterose of Black Soldier Fly (Hermetia illucens) Induces Innate Immune Response through Toll-Like Receptor Pathway in Mouse Macrophage RAW264.7 Cells[J]. Biomolecules, 2019, 9(11).

[13] Wang W , Zhong M , Yu T, et al. Polysaccharide extracted from WuGuChong reduces high-fat diet-induced obesity in mice by regulating the composition of intestinal microbiota[J]. Nutrition \& Metabolism, 2020, 17(1).

[14] Zong J , Jiang J , Shi P , et al. Fatty acid extracts facilitate cutaneous wound healing through activating AKT, ERK, and TGF- $\beta /$ Smad 3 signaling and promoting angiogenesis[J]. American Journal of Translational Research, 2020, 12(2):478-492.

[15] Xie, Song-Zi, Ji-Chun, et al. Digestive behavior of Dendrobium huoshanense polysaccharides in the gastrointestinal tracts of mice[J]. International Journal of Biological Macromolecules: Structure, Function and Interactions, 2018, 107(Pt.A):825-832.

[16] Zuo, T, Li, et al. Dietary fucoidan of Acaudina molpadioides and its enzymatically degraded fragments could prevent intestinal mucositis induced by chemotherapy in 
mice[J]. Food \& Function, 2015.

[17] Ali J , Khan A U , Shah F A, et al. Mucoprotective effects of Saikosaponin-A in 5-fluorouracil-induced intestinal mucositis in mice model[J]. Life Sciences, 2019, 239:116888-.

[18] Sakai H , Sagara A , Matsumoto K, et al. Neutrophil recruitment is critical for 5-fluorouracil-induced diarrhea and the decrease in aquaporins in the colon[J]. Pharmacological Research, 2014, 87(9):71-79.

[19] Galdino F , Andrade M , Barros P, et al. Pretreatment and treatment with fructo-oligosaccharides attenuate intestinal mucositis induced by 5 -FU in mice[J]. Journal of Functional Foods, 2018.

[20] Structure-function relationships of immunostimulatory polysaccharides: A review.[J]. Carbohydrate Polymers, 2015, 132:378-396.

[21] Xie, Song-Zi, Ge, et al. Digestive behavior of Dendrobium huoshanense polysaccharides in the gastrointestinal tracts of mice[J]. International Journal of Biological Macromolecules Structure Function \& Interactions, 2018.

[22] Rice, P. J . Oral delivery and gastrointestinal absorption of soluble glucans stimulate increased resistance to infectious challenge.[J]. Journal of Pharmacology \& Experimental Therapeutics, 2005, 314(3):1079-1086.

[23] Zhang S , Y Liu, D Xiang, et al. Assessment of dose-response relationship of 5fluorouracil to murine intestinal injury[J]. Biomedicine \& pharmacotherapy $=$ Biomedecine \& pharmacotherapie, 2018, 106:910-916.

[24] Ali J , Khan A U, Shah F A, et al. Mucoprotective effects of Saikosaponin-A in 5fluorouracil-induced intestinal mucositis in mice model[J]. Life Sciences, 2019, 239:116888-.

[25]Rebecca, Green, Heather, et al. Eating Experiences of Children and Adolescents With Chemotherapy-Related Nausea and Mucositis[J]. Journal of Pediatric Oncology Nursing, 2010, 27(4).

[26] Smith J L , Malinauskas B M , Garner K J , et al. Factors contributing to weight loss, nutrition-related concerns and advice received by adults undergoing cancer treatment.[J]. Adv Med, 2008, 53(2):198-204.

[27] Shi Y, Zhu H , Li R, et al. Effect of polysaccharides from Sijunzi decoction on $\mathrm{Ca} 2+$ related regulators during intestinal mucosal restitution[J]. Phytomedicine, 2019. [28] Bajic J E , E De N G L , Lampton L S , et al. Rhubarb extract partially improves mucosal integrity in chemotherapy-induced intestinal mucositis[J]. 世界胃肠病学杂 志: 英文版, 2016(37):12.

[29] Li J , Li R X , Liu G, et al. Effect of melatonin on renewal of chicken small intestinal mucosa[J]. Poultry Science, 2017, 96(8).

[30] Mccauley H A， Guasch G . Three cheers for the goblet cell: maintaining homeostasis in mucosal epithelia[J]. Trends in Molecular Medicine, 2015, 21(8):492-503.

[31] Luo Q , Lao C , Huang C , et al. Iron Overload Resulting from the Chronic Oral Administration of Ferric Citrate Impairs Intestinal Immune and Barrier in Mice[J]. Biological Trace Element Research, 2020(10).

[32] Pierre J F , Heneghan A F , Feliciano R P , et al. Cranberry Proanthocyanidins 
Improve Intestinal sIgA During Elemental Enteral Nutrition[J]. Jpen J Parenter Enteral Nutr, 2014, 38(1):107-114.

[33] Jing J X, Zhao Y L, Qin Y, et al. Evaluating Intestinal Permeability by Measuring Plasma Endotoxin and Diamine Oxidase in Children with Acute Lymphoblastic Leukemia Treated with High-dose Methotrexate[J]. Anti-Cancer Agents in Medicinal Chemistry (Formerly Current Medicinal Chemistry - Anti-Cancer Agents), 2016, 16(3):-.

[34] Yusuke H, Hiroshi N , Minoru M, et al. Clinical significance of serum diamine oxidase activity in inflammatory bowel disease[J]. Inflammatory Bowel Diseases, 2011(2):2.

[35] Yusuke H, Hiroshi N , Minoru M, et al. Clinical significance of serum diamine oxidase activity in inflammatory bowel disease[J]. Inflammatory Bowel Diseases, 2011(2):2.

[36]Conklin, K. A . Chemotherapy-Associated Oxidative Stress: Impact on Chemotherapeutic Effectiveness[J]. Integrative Cancer Therapies, 2004, 3(4):294-300. [37] Scalise A A, Kakogiannos N , Zanardi F , et al. The blood-brain and gut-vascular barriers: from the perspective of claudins[J]. 2021.

[38] Barmeyer C, Schulzke J D , Fromm M . Claudin-related intestinal diseases[J]. Seminars in Cell and Developmental Biology, 2015, 42:30-38.

[39] Iwamoto D V , Calderwood D A . Regulation of integrin-mediated adhesions[J]. Current opinion in cell biology, 2015, 36(6):41-47.

[40] Lameris A L , Huybers S , Kaukinen K , et al. Expression profiling of claudins in the human gastrointestinal tract in health and during inflammatory bowel disease[J]. Scandinavian Journal of Gastroenterology, 2013, 48(1):58.

[41] Alizadeh A, Akbari P, Garssen J, Fink-Gremmels J, Braber S. Epithelial integrity, junctional complexes, and biomarkers associated with intestinal functions. Tissue Barriers. 2021 Oct 30:1996830.

[42] Wu J , He C, Bu J , et al. Betaine attenuates LPS-induced downregulation of Occludin and Claudin-1 and restores intestinal barrier function. 2020.

[43] Youmba S B , Belmonte L, Galas L, et al. Methotrexate modulates tight junctions through NF-kB, MEK, and JNK pathways.[J]. Journal of Pediatric Gastroenterology \& Nutrition, 2012, 54(4):463-470.

[44]Vinícius da Silva Duarte, Cruz B , Tarrah A, et al. Chemoprevention of DMHInduced Early Colon Carcinogenesis in Male BALB/c Mice by Administration of Lactobacillus Paracasei DTA81[J]. Microorganisms, 8(12):1994.

[45] O"Flaherty S, Saulnier D , Pot B , et al. How can probiotics and prebiotics impact mucosal immunity?[J]. Gut Microbes, 2010, 1(5):293-300.

[46]Sonis, Stephen T . The pathobiology of mucositis.[J]. Nature Reviews Cancer, 2004, 4(4):277-84.

[47] Sonis S T. Sonis, S. T. The biologic role for nuclear factor- $\kappa \mathrm{B}$ in disease and its potential involvement in mucosal injury associated with anti-neoplastic therapy. Crit. Rev. Oral Biol. Med[J]. Critical Reviews in Oral Biology \& Medicine, 2002, 13(5):380389.

[48] A K N , Pr emila Abraham a, B R K, et al. NF- $\kappa B-i N O S-C O X 2-T N F \alpha$ 
inflammatory signaling pathway plays an important role in methotrexate induced small intestinal injury in rats[J]. Food and Chemical Toxicology, 2018, 118:766-783.

[49] Hotz-Behofsits C , Simpson R J , Walley M, et al. Role of COX-2 in nonsteroidal anti-inflammatory drug enteropathy in rodents[J]. Scandinavian Journal of Gastroenterology, 2010, 45(7-8):822.

[50] Aga B , Asa B , Esa B , et al. Tumor necrosis factor-alpha and interferon-gamma induce inflammasome-mediated corneal endothelial cell death[J]. Experimental Eye Research, 207.

[51] Al-Dasooqi N, Wardill H R, Gibson R J . Gastrointestinal Mucositis: The Role of MMP-Tight Junction Interactions in Tissue Injury[J]. Pathology \& Oncology Research, 2014, 20(3):485-491.

[52] Xu X , Xu P, Ma C , et al. Gut microbiota, host health, and polysaccharides[J]. Biotechnology Advances, 2013, 31(2):318-337.

[53] Martens E C , Koropatkin N M , Smith T J , et al. Complex Glycan Catabolism by the Human Gut Microbiota: The Bacteroidetes Sus-like Paradigm[J]. Journal of Biological Chemistry, 2009, 284(37):24673.

[54]Gentile CL, Weir TL. The gut microbiota at the intersection of diet and human health. Science. 2018 Nov 16;362(6416):776-780. doi: 10.1126/science.aau5812. PMID: 30442802.

[55] Ferreira T M , Leonel A J , Melo M A, et al. Oral Supplementation of Butyrate Reduces Mucositis and Intestinal Permeability Associated with 5-Fluorouracil Administration[J]. LIPIDS -CHICAGO THEN CHAMPAIGN- AMERICAN OIL CHEMISTS SOCIETY, 2012, 47(7):669-678.

[56]Flávia Mendes Peradeles Galdino a, Maria Emília Rabelo Andrade a, Patrícia Aparecida Vieira de Barros b, et al. Pretreatment and treatment with fructooligosaccharides attenuate intestinal mucositis induced by $5-\mathrm{FU}$ in mice[J]. Journal of Functional Foods, 2018, 49:485-492.

[57]Peilin Chen, Suzhen Lei, Mingyao Tong, Qing Chang, Baodong Zheng, Yi Zhang, Hongliang Zeng,Effect of polysaccharide fractions from Fortunella margarita on the fecal microbiota of mice and SCFA production in vitro,Food Science and Human Wellness, Volume 11, Issue 1,2022,Pages 97-108,ISSN 2213-4530

[58] Khan I, Huang G , Li X, et al. Mushroom polysaccharides from Ganoderma lucidum and Poria cocos reveal prebiotic functions[J]. Journal of Functional Foods, 2018, 41:191-201.

[59] Shi H , Chang Y, Yuan G, et al. Dietary fucoidan of Acaudina molpadioides alters gut microbiota and mitigates intestinal mucosal injury induced by cyclophosphamide.[J]. Food \& Function, 2017, 8(9):3383-3393. 